\title{
Zero-variance principle for Monte Carlo algorithms
}

\author{
Roland Assaraf and Michel Caffarel \\ CNRS-Laboratoire de Chimie Théorique Tour 22-23, Université Pierre et Marie Curie 4 place \\ Jussieu \\ 75252 Paris Cedex 05; France e-mail: ra@lct.jusssieu.fr and mc@lct.jusssieu.fr
}

We present a general approach to greatly increase at little cost the efficiency of Monte Carlo algorithms. To each observable to be computed we associate a renormalized observable (improved estimator) having the same average but a different variance. By writing down the zero-variance condition a fundamental equation determining the optimal choice for the renormalized observable is derived (zerovariance principle for each observable separately). We show, with several examples including classical and quantum Monte Carlo calculations, that the method can be very powerful.

PACS No:02.70.Lq,05.10.Ln,05.50.+q,75.40.Mg 
Since the pioneering work of Metropolis et al. [1] Monte Carlo methods have been widely used in many areas of natural sciences. At the root of Monte Carlo methods lies a very efficient stochastic method for calculating many-dimensional integrals (or sums) written under the general form

$$
<O>\equiv \frac{\int_{S} d x \pi(x) O(x)}{\int_{S} d x \pi(x)}
$$

where $O(x)$ is some arbitrary observable (real-valued function) defined on the configuration space $S$ (continuous or discrete) and $\pi(x)$ some probability distribution. In Monte Carlo methods the integrals are evaluated using a large but finite set of configurations $\left\{x^{(i)}\right\}_{i=1, N}$ distributed according to $\pi$ and generated by a step-by-step stochastic procedure (Markov chain)

$$
<O>=\frac{1}{N} \sum_{i=1}^{N} O\left[x^{(i)}\right]+\delta O,
$$

where $\delta O$ is the statistical error associated with the finite statistics. For a large enough number $N$ of Monte Carlo steps, standard statistical arguments lead to the following expression of the error

$$
\delta O=K \frac{\sigma(O)}{\sqrt{N}}
$$

where $K$ is some positive constant proportional to the amount of correlation between configurations and $\sigma(O)$ a measure of the fluctuations of the observable

$$
\sigma(O) \equiv \sqrt{\left.<O^{2}>-<O\right\rangle^{2}} .
$$

In this Letter it is shown that by introducing a suitably renormalized observable $\tilde{O}(x)$ the statistical error can be drastically reduced and even suppressed, thus defining a zerovariance principle for the Monte Carlo calculation of observables. To realize this, a trial operator $H$ and a trial function $\psi(x)$ are introduced (a trial matrix and a trial vector in the discrete case). The operator $H$ is supposed to be Hermitian (in all practical applications, real symmetric) and is chosen such that

$$
\int d y H(x, y) \sqrt{\pi(y)}=0 .
$$

On the other hand, the trial function $\psi(x)$ is a rather arbitrary function which is simply supposed to be integrable. Now, the renormalized observable $\tilde{O}(x)$ associated with the observable $O(x)$ is defined as follows

$$
\tilde{O}(x)=O(x)+\frac{\int d y H(x, y) \psi(y)}{\sqrt{\pi(x)}} .
$$

As a direct consequence of Eq. (1) and of the very definition of the Hermitian operator $H$, Eq. (5), we have the important property 


$$
<\tilde{O}>=<O>.
$$

In other words, both quantities $O(x)$ and $\tilde{O}(x)$ can be used as estimators of the desired average. However, the statistical errors, which are controlled by $\sigma(O)$ and $\sigma(\tilde{O})$, can be very different. The optimal choice for $(H, \psi)$ is obtained by imposing the renormalized function to be constant and equal to the exact average. This leads to the following fundamental equation

$$
\int d y H(x, y) \psi(y)=-[O(x)-<O>] \sqrt{\pi(x)} \Leftrightarrow \sigma(\tilde{O})=0
$$

At this point it should be emphasized that the idea of using renormalized estimators for reducing the variance is not new. A number of applications have been performed using various "improved" estimators having a lower variance (See, e.g., [2], [3]). The basic idea is to construct new estimators by integrating out some intermediate degrees of freedom and, therefore, removing the corresponding source of fluctuations. However, to the best of our knowledge, no general and systematic approach based on a zero-variance principle and valid for any type of Monte Carlo methods has been proposed so far.

In this work the following strategy is proposed. First, a Hermitian operator $H$ verifying (5) is chosen. Second, some approximate solution of Eq.(8) is searched for. The various parameters entering $\psi$ are then optimized by minimizing the fluctuations of the renormalized observable over a finite set of points distributed according to $\pi$ and obtained from a short Monte Carlo calculation. Finally, a standard much longer Monte Carlo simulation is performed using $\tilde{O}(x)$ instead of $O(x)$ as estimator.

Choice of $H$. Clearly, a large variety of choices are possible for the trial operator $H$. For Monte Carlo algorithms satisfying the detailed balance condition (in practice, the vast majority of MC schemes) a very natural choice is at our disposal. Denoting $p(x \rightarrow y)$ the transition probability distribution defining the Monte Carlo dynamics, the detailed balance condition is written as $\pi(x) p(x \rightarrow y)=\pi(y) p(y \rightarrow x)$ for all pairs $(\mathrm{x}, \mathrm{y})$ in configuration space. A most natural operator to consider is

$$
H(x, y)=\sqrt{\frac{\pi(x)}{\pi(y)}}[p(x \rightarrow y)-\delta(x-y)] .
$$

From the detailed balance condition it follows that the operator $H$ is symmetric, $H(x, y)=$ $H(y, x)$. The fundamental property (5) is verified since the sum-over-final-states for a transition probability is equal to one. For continuous systems Schroedinger-type Hamiltonians can also be considered

$$
H=-\frac{1}{2} \sum_{i=1}^{d} \frac{\partial^{2}}{\partial x_{i}{ }^{2}}+V(x)
$$

where $V(x)$ is some local potential constructed to fulfill condition (5):

$$
V(x)=\frac{1}{2 \sqrt{\pi(x)}} \sum_{i=1}^{d} \frac{\partial^{2} \sqrt{\pi(x)}}{\partial x_{i}{ }^{2}},
$$


where $d$ is the number of degrees of freedom. Note that in Eq. (10) $H$ is written using the standard quantum-mechanical notation for a local Hamiltonian in the $x$-space realization.

Choice of $\psi$. Once the operator $H$ has been chosen, the optimal choice for $\psi$ is the exact solution of the fundamental equation. Of course, in practice only approximate solutions are available. What particular form to choose for $\psi$ is very dependent on the problem at hand, on the type of observables considered, and also on the form chosen for the trial operator $H$. However, a most important point to be stressed is that the global normalization factor associated with $\psi$ is a pertinent parameter of the trial function. Minimizing the fluctuations of the renormalized function $\sigma(\tilde{O})$ with respect to it, we get

$$
\sigma(\tilde{O})^{2}=\sigma(O)^{2}-\frac{<\frac{O(x) \int d y H(x, y) \psi(y)}{\sqrt{\pi(x)}}>^{2}}{<\left(\frac{\int d y H(x, y) \psi(y)}{\sqrt{\pi(x)}}\right)^{2}>} .
$$

The correction to $\sigma(O)^{2}$ being negative we obtain the important result that, whatever the choice made for the trial function (even the most unphysical one!), the optimization of the multiplicative factor always leads to a reduction of the statistical error.

Our first application concerns the Monte Carlo calculation of the internal energy of the standard $2 D$-Ising model at various temperatures and linear sizes $L=5,10,20$, and 25 . The observable considered is the energy function given by $E(S)=-\sum_{<i, j>} S_{i} S_{j}$ (coupling constant $J=1$, sum limited to nearest neighbors, and periodic boundary conditions). The probability distribution is $\pi(S)=\exp [-\beta E(S)]$ with $\beta=1 / k_{B} T$. Here, $S \equiv\left(S_{1}, \cdots, S_{N}\right)$ with $S_{i}= \pm 1$, and $N=L \times L$ is the total number of spins. Simulations have been performed using a Swendsen-Wang type algorithm [4](non-local updates of clusters of spins). To construct the trial operator $H$ we have chosen to use the transition probability distribution of Monte Carlo algorithms with local updates ("Heat-Bath"-type algorithms). The probability of flipping the spin $S_{i}= \pm 1$ at site $i$ is given by

$$
p\left(S_{i} \rightarrow \epsilon S_{i}\right)=\frac{e^{\beta \epsilon S_{i} \tilde{S}_{i}}}{e^{\beta S_{i} \tilde{S}_{i}}+e^{-\beta S_{i} \tilde{S}_{i}}}
$$

where $\epsilon=1$ (no flip) or -1 (flip), and $\tilde{S}_{i}$ is the sum of neighboring spin values. With this choice and using Eq.(9) the fundamental equation (8) can be rewritten under the form

$$
\begin{gathered}
\sum_{i=1}^{N} p\left(S \rightarrow T_{i} S\right)\left[Q(S)-Q\left(T_{i} S\right)\right]=E(S)-<E> \\
\psi(S)=Q(S) \sqrt{\pi(S)}
\end{gathered}
$$

where the application $T_{i}(i=1, \cdots, N)$ describes a flip at site $i$ and is defined by $T_{i}\left(S_{1}, \cdots, S_{i}, \cdots, S_{N}\right)=\left(S_{1}, \cdots,-S_{i}, \cdots, S_{N}\right)$. At $\beta=0(T=\infty)$ the transition probability distribution becomes constant and the exact solution is easily found to be $Q(S)=E(S) / 2$. For finite temperatures some approximate solution has to be found. Here we introduce for $Q(S)$ a polynomial expansion up to the fourth-order in the variables $X=\sum_{i=1}^{N} S_{i}$ (magnetization) and $Y=\sum_{i=1}^{N} g\left(S_{i} \tilde{S}_{i}\right)$ ("generalized energy", the usual energy being recovered 
for $g(x)=x)$. Precisely, we have chosen the form $Q(S)=e^{-Z} \sum_{n+m \leq 4} c_{n m} X^{n} Y^{m}$ where $Z=\sum_{i=1}^{N} h\left(S_{i} \tilde{S}_{i}\right)$. The set of variational parameters of $\psi$ consists of all coefficients $c_{n m}$ of the polynomial plus the ten possible values of functions $g$ and $h$. All coefficients have been optimized by minimizing the fluctuations of the renormalized energy $\sigma(\tilde{E})$ defined by (田) and calculated from 2000 to 5000 different spin configurations $S^{(i)}$ drawn according to $\pi$. Finally, the last step consists in performing a long Monte Carlo simulation to compute accurately the various quantities. The number of clusters built varies from $10^{6}$ (for the larger size) to $2.10^{8}$ (for the smaller size). Results are presented in Table $\mathbb{E}$. Three different temperatures have been considered. $T=3$ corresponds to the low-temperature regime, $T=T_{c}=4 / \ln (\sqrt{2}+1)$ is the critical temperature for the infinite lattice, and $T=8$ is in the high-temperature regime of the model. At $T=3$, our representation is extremely good whatever the size of the lattice considered. The variance associated with the renormalized energy is drastically reduced with respect to the bare value and the gain in computational effort can be as great as $\sim 360$. Here, the gain in computational effort is defined as the ratio of the squared statistical errors, $(\delta \tilde{E} / \delta E)^{2}$. In other words, according to Eq.(3) it represents the factor by which it would be necessary to increase the number of Monte Carlo steps in the standard approach to get the same accuracy. Note that for $L=5$ our Monte Carlo value coincides with the exact one (computed by exact numeration of the $2^{N}$ configurations) with an accuracy of less than $10^{-6}$. Note also that our MC values converge as the size is increased to the exact infinite-lattice value as given by the Onsager solution [5]. At $T=8$ (high-temperature regime) our representation is less good but still very satisfactory. As a function of the size, the gain in computational effort converges and a value of about 20 is gotten. At the infinite-lattice critical value the results are less spectaculary but still of interest. A converged value of about 3 for the gain in efficiency is obtained. At this temperature the correlation length for the spin variables diverges and more accurate representations for the solution of Eq.(14) are needed. Starting from our basic equation built from a transition probability corresponding to local moves we need to resort to approximate solutions which contain in some way the collective spin excitations. Alternatively, we can change our fundamental equation by resorting to a non-local transition probability density and, then, to a new operator $H$. A natural choice is of course the transition probability of the Swendsen-Wang algorithm used here to generate configurations. Preliminary calculations show that statistical fluctuations are indeed strongly decreased. However, to sum up analytically all contributions corresponding to the different Swendsen-Wang clusters (action of $H$ on $\psi$ ) is very time-consuming and the advantages of the method can be lost. Some approximate scheme is clearly called for; this is let for future development. Finally, a last important point is that the gain in computational effort is found to be systematically greater (by about 50\%) than the corresponding ratio of variances. This result is a direct consequence of the fact that the integrated autocorrelation time known to control the amount of correlation between successive measurements (see, e.g., [3]) has been decreased when passing from the bare observable to the renormalized one. Note that a similar behavior has also been obtained in applications based on improved estimators [2], [3]. Without entering into the details, it can be shown that this result is directly related to the fact that the fluctuations of the renormalized observable are much smaller than in the bare case.

The second application illustrates the method in the case of a continuous configuration space (calculation of multi-dimensional integrals). We have calculated a mean energy as 
it appears in the so-called Variational Monte Carlo (VMC) methods [6]. Starting from a quantum Hamiltonian $H_{Q}$ (to be distinguished from our trial operator $H$ ) and a known trialwave function $\psi_{T}$ our purpose is to compute the variational energy $E_{v}$ associated with $\psi_{T}$. $E_{v}$ can be easily rewritten as an average over the probability distribution $\psi_{T}^{2}, E_{v}=\left\langle E_{L}>\right.$ where $E_{L} \equiv H_{Q} \psi_{T} / \psi_{T}$ is called the local energy. Here, we consider the case of the Helium atom described by the Hamiltonian $H_{Q}=-1 / 2\left(\vec{\nabla}_{1}^{2}+\vec{\nabla}_{2}^{2}\right)-2 / r_{1}-2 / r_{2}+1 / r_{12}$ (atomic units) with usual notations. As trial wave function a standard form has been chosen [7]

$$
\psi_{T}\left(\vec{r}_{1}, \vec{r}_{2}\right)=\exp \left[\frac{a r_{12}}{1+b r_{12}}-c\left(r_{1}+r_{2}\right)\right] 1 s\left(r_{1}\right) 1 s\left(r_{2}\right)
$$

where $1 s(r)$ is the Hartree-Fock orbital as given by Clementi and Roetti [\$] and the variational parameters have been chosen to be $a=0.5, b=0.522$, and $c=0.0706$. As already remarked a natural choice for the trial Hamiltonian $H$ is a Schroedinger operator admitting $\psi_{T}$ as ground-state, Eqs. (10,11). Regarding $\psi$ we have chosen a form similar to the trial wavefunction multiplied by some function of the potential energy. Configurations are generated using a standard Metropolis algorithm with local moves constructed using a Langevin equation [7]. Results are presented in Table II. It is seen that the introduction of the renormalized local energy increases the efficiency of the Monte Carlo calculation by about one order of magnitude.

In the last application it is shown that the method can even be used in exact (zerotemperature) quantum Monte Carlo (QMC) calculations. In QMC a combination of diffusion and branching process is used to construct a stationary density proportional to $\psi_{T} \psi_{0}$ where $\psi_{0}$ is the exact unknown ground-state wavefunction. By averaging the local energy over this distribution an estimate of the exact energy $E_{0}$ is obtained [6]. Although the analytical form of the stationary density is no longer known, a renormalized function whose average is identical to that of the bare local energy can still be defined, $\tilde{E}_{L}=E_{L}+\left(H-E_{0}\right) \psi / \psi_{T}$, where $H$ admits $\psi_{T}$ as eigenvector, $H \psi_{T}=0$. Calculations have been done using the exact Green's function Monte Carlo of Ceperley and Alder [9]. Results are presented in Table [1. They are of a quality similar to that obtained in the variational case. About one order of magnitude in computer time has been gained.

To conclude we have presented a simple and powerful method to greatly increase at little cost the efficiency of Monte Carlo calculations. The examples presented have been chosen to illustrate the great versatility of the method (discrete and continuous configuration spaces, classical or quantum Monte Carlo, local or non-local Monte Carlo updates). Although our examples have only been concerned with total energies, let us emphasize that the zerovariance principle is valid for any type of observable including important quantities such as local properties other than energy, differences of energies, spatial correlation functions, etc... 


\section{REFERENCES}

[1] N. Metropolis, A.W. Rosenbluth, M.N. Rosenbluth, A.H Teller, and E. Teller, J. Chem. Phys. 21, 1097 (1953).

[2] M. Sweeny, Phys. Rev. B 27, 4445 (1983); G. Parisi, R. Petronzio, and F. Rapuano, Phys. Lett. B 128, 418 (1983); U. Wolff, Nucl. Phys. B 334, 581 (1990)

[3] B. Ammon, H.G. Evertz, N. Kawashima, M. Troyer, and B. Frischmuth, Phys. Rev. B 58, 4304 (1998).

[4] R.H. Swendsen and J.S. Wang, Phys. Rev. Lett. 58, 86 (1987).

[5] L. Onsager, Phys. Rev. 65, 117 (1944).

[6] W. von der Linden, Phys. Rep. 220, 53 (1992).

[7] K.E Schmidt and J.W. Moskowitz, J. Chem. Phys. 93, 4172 (1990).

[8] E. Clementi and C. Roetti, Atomic Data and Nuclear Data Tables 14, 177(1974)

[9] D.M. Ceperley and B.J. Alder, J. Chem. Phys. 64, 5833 (1984).

[10] K. Frankowski and C.L. Pekeris, Phys. Rev. 146, 46 (1966). 


\section{TABLES}

TABLE I. Internal Energies for the 2D-Ising model at different temperatures. $N$ number of sites. Statistical uncertainties on the last digit are indicated in parentheses.

\begin{tabular}{|c|c|c|c|c|c|}
\hline$\overline{\text { Size }}$ & $5 \times 5$ & $10 \times 10$ & $20 \times 20$ & $25 \times 25$ & $\infty \times \infty$ \\
\hline \multicolumn{6}{|l|}{$\overline{\bar{T}=3}$} \\
\hline$\overline{\sigma(E)^{2} / N}$ & 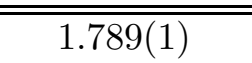 & $\overline{1.777(2)}$ & $\overline{~ 1.78(1)}$ & $\overline{~ 1.79(1)}$ & \\
\hline$\sigma(\tilde{E})^{2} / N$ & $0.0125(4)$ & $0.0061(1)$ & $0.0060(2)$ & $0.0061(2)$ & \\
\hline Ratio of variances & $\sim 143$ & $\sim 291$ & $\sim 297$ & $\sim 293$ & \\
\hline$<E / N>$ & $-3.902044(31)$ & $-3.902200(55)$ & $-3.90217(21.4)$ & $-3.90242(29)$ & \\
\hline$<\tilde{E} / N>$ & $-3.902020(2.4)$ & $-3.902229(3)$ & $-3.90225(1.2)$ & $-3.90222(1.5)$ & \\
\hline Gain in computational effort ${ }^{a}$ & $\sim 167$ & $\sim 336$ & $\sim 318$ & $\sim 360$ & \\
\hline$\leq E / N>$ Exact value & $-3.9020214 \ldots$ & & & & -3.9022331 \\
\hline \multicolumn{6}{|l|}{$\bar{T}=T_{c}=4.53837 .}$. \\
\hline$\overline{\overline{\sigma(E)^{2} / N}}$ & $\overline{18.581(4)}$ & $25.97(3)$ & $33.1(2)$ & $35.3(2)$ & \\
\hline$\sigma(\tilde{E})^{2} / N$ & $0.215(2)$ & $4.85(1)$ & $16.5(1)$ & $16.9(2)$ & \\
\hline Ratio of variances & $\sim 86$ & $\sim 5.4$ & $\sim 2.0$ & $\sim 2.1$ & \\
\hline$<E / N>$ & $-3.07334(13)$ & $-2.95214(33)$ & $-2.8902(12)$ & $-2.8800(14)$ & \\
\hline$<\tilde{E} / N>$ & $-3.07345(1.3)$ & $-2.95236(13)$ & $-2.8908(7)$ & $-2.8788(8)$ & \\
\hline Gain in computational effort ${ }^{a}$ & $\sim 100$ & $\sim 6.8$ & $\sim 3$ & $\sim 3.1$ & \\
\hline$\leq E / N>$ Exact value & $-3.0734396 \ldots$ & & & & -2.8284271. \\
\hline \multicolumn{6}{|l|}{$\overline{T=8}$} \\
\hline$\overline{\sigma \sigma(E)^{2} / N}$ & $\overline{13.17(1)}$ & 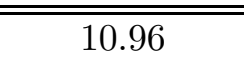 & 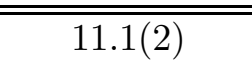 & 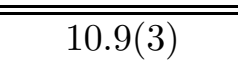 & \\
\hline$\sigma(\tilde{E})^{2} / N$ & 0.041 & 0.455 & $0.8(1)$ & $0.9(1)$ & \\
\hline Ratio of variances & $\sim 321.2$ & $\sim 24$ & $\sim 13.9$ & $\sim 12$ & \\
\hline$<E / N>$ & $-1.16440(33)$ & $-1.11556(48)$ & $-1.1156(20)$ & $-1.1165(25.6)$ & \\
\hline$<\tilde{E} / N>$ & $-1.16348(1.6)$ & $-1.11502(8.2)$ & $-1.1145(4.4)$ & $-1.1145(5.6)$ & \\
\hline Gain in computational effort ${ }^{a}$ & $\sim 425$ & $\sim 34$ & $\sim 20.7$ & $\sim 20.9$ & \\
\hline$\leq E / N>$ Exact value & $-1.1634926 \ldots$ & & & & -1.1145444 . \\
\hline
\end{tabular}

a See text for definition.

${ }^{b}$ Ref. [5]. 
TABLE II. Energy of the Helium atom. All quantities are given in atomic units. Statistical uncertainties on the last digit are indicated in parentheses.

\begin{tabular}{|c|c|}
\hline \multicolumn{2}{|l|}{ Variational Monte Carlo } \\
\hline$\overline{\overline{\sigma\left(E_{L}\right)^{2}}}$ & $0.0409(2)$ \\
\hline$\sigma\left(\tilde{E}_{L}\right)^{2}$ & 0.00688 \\
\hline Ratio of variances & $\sim 5.9$ \\
\hline$<E_{L}>$ & $-2.89671(4.8)$ \\
\hline$<\tilde{E}_{L}>$ & $-2.89674(1.6)$ \\
\hline Gain in computational effort ${ }^{a}$ & $\sim 9$ \\
\hline \multicolumn{2}{|c|}{$\begin{array}{l}\text { Exact Green's Function Monte Carlo } \\
\end{array}$} \\
\hline$\overline{\overline{\sigma\left(E_{L}\right)^{2}}}$ & $0.0411(9)$ \\
\hline$\sigma\left(\tilde{E}_{L}\right)^{2}$ & $0.00855(8)$ \\
\hline Ratio of variances & $\sim 4.9$ \\
\hline$<E_{L}>$ & $-2.903745(99)$ \\
\hline$<\tilde{E}_{L}>$ & $-2.903734(33)$ \\
\hline Gain in computational effort ${ }^{a}$ & $\sim 9$ \\
\hline Exact energy & $-2.903724377 \ldots{ }^{c}$ \\
\hline
\end{tabular}

$\overline{\bar{a} \text { See text for definition. }}$

${ }^{b}$ Reference [9]

${ }^{c}$ Reference 10 